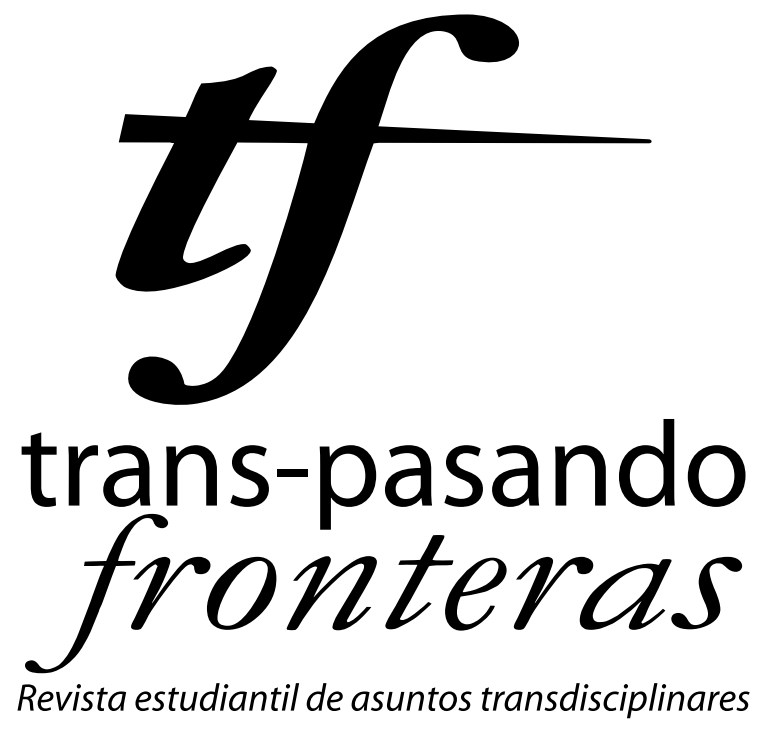

Una publicación de

Facultad de Derecho y Ciencias Sociales

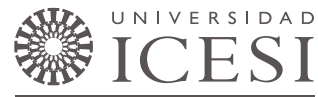




\title{
La Ley 70 de 1993 y la participación política de las comunidades negras"
}

\author{
Alejandra Erazo Gómez \\ (aleja.erazo@hotmail.com)
}

Artículo de investigación científica y tecnológica recibido el 28/08/2012 y aprobado el 17/10/2012.

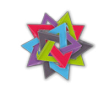

Como citar este artículo:

ERAZO G. Alejandra (2012). "La Ley 70 de 1993 y la participación política de las comunidades negras". En: Trans-pasando Fronteras, Núm. 2, pp. 31-45. Cali, Colombia: Centro de Estudios Interdisciplinarios, Jurídicos, Sociales y Humanistas (CIES), Universidad Icesi.

\begin{abstract}
Resumen
El siguiente documento se elaboró como una reflexión en el marco de las dos décadas de promulgación de la Constitución de 1991, la cual enuncia el carácter multicultural y pluriétnico de la nación colombiana. Nuestra Carta Política se inscribe en la ola del multiculturalismo que se institucionalizó justamente en esa década como la forma hegemónica de pensar las diferencias culturales. Este artículo quiere recoger los planteamientos sobre participación política de grupos afrocolombianos consagrados en la Constitución de 1991 y en la Ley 70 de 1993, proponiendo un ejercicio de confrontación con las prácticas reales de participación de estos grupos. Para éste propósito el ensayo se estructura en tres partes. La primera presenta los fundamentos teóricos del multiculturalismo, acción política y el contexto de modernidad. La segunda parte señala algunos antecedentes apropósito de la participación política de estas comunidades. Y en la tercera se hace referencia a las condiciones actuales de participación resaltando los problemas y dificultades que han experimentado.
\end{abstract}

* $\quad$ Artículo ganador de la segunda versión del Concurso de Ensayo Político de la Universidad Icesi. El tema de la convocatoria fue: "Dos décadas de la Constitución Política de 1991". 
Palabras claves:

Multiculturalismo; Comunidades negras; Ley 70; Participación política

\section{Introducción}

En 1991 promulgó la actual Constitución Política de Colombia que reemplazó a la de 1886 después de más de un siglo de vigencia. Entre las muchas continuidades y rupturas frente a su antecesora, la actual Carta Magna dio un giro importante al institucionalizar el carácter pluriétnico y multicultural del país, contrastando con el discurso de nación mestiza esgrimido durante gran parte del siglo pasado. Además, se pasó de una democracia representativa a una participativa, consagrando mecanismos diferentes al voto para garantizar la participación ciudadana en asuntos económicos, políticos, administrativos y culturales del país. La conjugación de estos dos aspectos es precisamente el tema que me interesa abordar en las siguientes páginas. Me refiero concretamente a la articulación del reconocimiento del multiculturalismo con la participación política de las minorías afrocolombianas.

Las propuestas relacionadas con la institucionalización del multiculturalismo y la visibilización de las minorías culturales, tenían, entre otros, el propósito de "facilitar la capacidad de las comunidades culturales de participar en la vida politica nacional, permitir el autogobierno de las minorías y dotarlas de instrumentos jurídicos para proteger y reproducir su legado cultural" (Bonilla, 2006: 125). Teniendo en cuenta las luchas sociales y políticas que han protagonizado grupos afrocolombianos en busca de la reivindicación de sus derechos y en contra de la discriminación racial, y a su vez, la representación indirecta que tuvieron en la Asamblea Nacional Constituyente, es pertinente intentar contrastar lo que se ha dicho y lo que se ha hecho acerca de estos respectos, en el marco de las dos décadas de la Constitución de 1991.

Es importante notar, además, que aunque la Carta Política haga énfasis en el multiculturalismo y la protección de la diferencia, el texto en sí se refiere explícita y casi únicamente a las comunidades indígenas como sujetos de la legislación. Sólo el Artículo Transitorio 55 -desarrollado luego por la Ley 70 de 1993- hace referencia manifiesta a las comunidades negras (rurales ribereñas) y a su derecho de apropiación colectiva de sus tierras ancestrales. De este modo se evidencia que el documento se construyó, res- 
pecto a reivindicaciones de derechos diferenciados de las comunidades étnicas, tomando al indígena como el referente constante de otredad (Agudelo, 2005). Lo anterior como resultado de circunstancias históricas sobre las que volveré más tarde.

El siguiente documento quiere recoger, entonces, los planteamientos sobre participación política de grupos afrocolombianos consagrados en la Constitución de 1991 y en la Ley 70 de 1993 y confrontarlos con las prácticas de participación de estos grupos. Para éste propósito el ensayo se estructurará en tres partes. La primera presentará brevemente y de manera no exhaustiva los fundamentos teóricos sobre multiculturalismo, acción política y el contexto de modernidad en el cual se inscriben estos movimientos. La segunda parte tratará, a manera de antecedentes, sobre la confluencia de actores alrededor de la gente afro, que llevó a la participación indirecta de estas comunidades en el desarrollo de la Asamblea Nacional Constituyente (ANC). La tercera parte hará referencia a las condiciones de participación de las comunidades negras, según lo consagrado en la Carta Política y en la Ley 70, resaltando los problemas y dificultades que se han presentado con tales disposiciones, especialmente lo relacionado con la representatividad de dichas comunidades.

Por último, cabe aclarar que este artículo no es resultado de una indagación empírica, sino de una revisión teórica y bibliográfica del tema enunciado. Para tal efecto me he apoyado consistentemente en el trabajo de Carlos Agudelo sobre multiculturalismo y poblaciones negras en Colombia, el cual se configuró como el principal referente teórico e histórico de la argumentación.

\section{Fundamentos teóricos}

En la Constitución de 1991 hay una tensión teórica y práctica -como lo evidencia Bonilla- entre dos tendencias que parecen opuestas, a saber: la igualdad y unidad política y la diferencia y autonomía política. En lugar de fijarse en uno de los dos extremos, la Carta se mueve pendularmente, oscilando entre la promulgación de la igualdad de todos los ciudadanos y el reconocimiento de la diferencia de los grupos étnicos, y entre el proyecto de unidad política y la autonomía territorial, política, económica y educativa de las comunidades indígenas (Bonilla, 2006). Sobre lo último, el proyecto de nación institucionalizado por la Constitución de 1886 privilegiaba la unidad política, que además, implicaba la unidad cul- 
tural en forma de homogenización. La fragmentación, la aceptación de singularidades, en lo referido por Wills, se considerada peligrosa para el bienestar nacional (Wills, 2000). Hoy, sin embargo, "el reconocimiento del multiculturalismo exige a los Estados democráticos liberales aceptar la diversidad de intereses e identificaciones morales emanadas de la heterogeneidad cultural” (Moreno, 2010). Además, y volviendo al primer antagonismo, "sólo vinculando diversidad con participación en condiciones de simetría se puede articular el derecho a la igualdad con el derecho a la diferencia” (Wills, 2000: 403).

Por su parte, los reclamos por el reconocimiento de la diferencia responden al fenómeno de descentramiento del sujeto dentro del contexto de la postmodernidad, que según Hall consiste en la pérdida del centro de las posiciones de identidad y el paso a la articulación de pertenencias múltiples y contradictorias (Hall, 2010). No obstante, como explica Gros para los indígenas, la reivindicación de su identidad cultural ante el Estado que pasa por el proceso de etnización de las comunidades, deviene en la creación de una identidad genérica, estática que contrasta con la variedad de identificaciones existentes (Gros, 2000). Este fenómeno es probablemente equiparable a la situación de las comunidades afrocolombianas, quienes para lograr la titulación colectiva de un terreno deben cumplir con ciertas condiciones explicitadas por la Ley $70^{1}$. Resalta, en especial, la falta de representación de las concentraciones en áreas urbanas, dónde se construyen posiciones de sujeto que no caben bajo lo estipulado por dicha legislación.

Es importante no perder de vista el papel que juega la participación política en las reivindicaciones, tanto de los sectores rurales como de los urbanos. Siguiendo a Agudelo, entiendo entonces la acción política como "las estrategias de competición entre grupos" (Agudelo, 2005: 101), en este caso entre la minoría afro y las fuerzas políticas hegemónicas y hasta entre las mismas minorías étnicas, por recursos materiales y lugares de poder. Por tal motivo, la "actividad fundamental de los actores politicos organizados colectivamente en partidos o movimientos (es justamente ésta) [...] búsqueda o conservación del poder" (Balandier citado en Agudelo, 2005:101). No obstante, también es importante aclarar que la participación política a la que me referiré en el caso de las comunidades negras, emana no sólo de los lugares de poder político institucional sino de iniciativas y organizaciones propias de las comunidades, como se verá más adelante.

I También referida como Ley de Comunidades Negras o Ley de Negritudes. 


\section{Antecedentes}

Como apunté antes, el negro, en contraste con el indígena, no era considerado como sujeto de otredad al que se debía reivindicar. Esto se puede deber, como señalan algunos autores, a que desde 1851, cuando se declaró la abolición de la esclavitud, los derechos de los afrocolombianos quedaron equiparados a los del resto de los ciudadanos (Agudelo, 2005; Sánchez, 1993); por tal motivo, aunque siguen siendo sujetos de exclusión y marginalidad, la 'deuda' histórica se considera saldada. De cualquier forma, como evidencia Agudelo, dicha legislación no representó verdaderas condiciones de igualdad pues aún se implementaban restricciones en el acceso a la educación y, por lo tanto, en los posibles oficios a realizar. De esta forma, según señalamiento del mismo autor, se conformó un orden social construido desde las élites, nutrido y sustentado por las teorías 'científicas' legadas de Europa (Agudelo, 2005: 39). O tal vez la huella del discurso del mestizaje obstaculiza la aceptación de reclamos de la diferencia étnico-racial que exigen las minorías discriminadas.

Agudelo introduce una idea clave para la comprensión de las dinámicas pasadas y presentes de participación de las comunidades negras en el ámbito nacional, diciendo que "el proceso histórico de la inserción de los pobladores negros en la sociedad nacional se ha movido entre la ambigüedad de la inclusión y la exclusión” (Agudelo, 2005: 61). Esto es, como sucedió en el siglo XVIII, "por una parte son reconocidos como "ciudadanos”, con algunas posibilidades de movilidad individual, por otra parte considerados como seres inferiores" (Agudelo, 2005: 40) o simplemente son invisibilizados.

En un plano general, son cuatro los factores a los que haré referencia para contextualizar las condiciones previas a la ANC, en miras de comprender la participación indirecta que tuvieran las negritudes y su futura construcción en la Ley 70. A saber: la Iglesia, el discurso de desarrollo, la descentralización y la filiación política tradicional. Lo anterior enfocado a su influencia en las condiciones que se vivían en Colombia y en la región del Pacífico en particular.

Como se sabe, la Iglesia tuvo una fuerte presencia como fuerza evangelizadora del 'otro' durante la Colonia. Sin embargo, Agudelo, entre otros autores, señala que su posición fue ambivalente en tanto abogaban por un trato más humano a los esclavos, más no se oponían a la esclavitud como tal (Agudelo, 2005: 46-47). En el Pacífico, el papel de dicha institución cambió a partir de 1970 cuando asumió el "impulso de procesos organizativos de las 
poblaciones" (Agudelo, 2005: 53), buscando la valoración y preservación de la 'identidad y cultura' afroamericanas. Esto iba acorde con las pretensiones proteccionistas y conservacionistas que rodeaban la otredad y que fueron promovidas por académicos a mediados del siglo XX. De la mano de la Iglesia estuvo el poder político -consolidado en el partido Conservador-y la determinación de los contenidos y lugares educativos del país. Con las restricciones del acceso a la educación, sea por segregación o falta de infraestructura, vienen también las limitaciones en la movilidad y ascenso social.

En cuanto al desarrollo y la descentralización, ambos son discursos pregonados por el neoliberalismo, institucionalizados luego en la Constitución de 1991. Sin embargo, tuvieron una presencia no desdeñable en las décadas precedentes. Sobre el desarrollo en el Pacífico, Agudelo señala que este buscaba un articulación de lo local y regional, tanto económica como de procesos culturales y políticos, con el ámbito nacional e internacional (Agudelo, 2005). Entonces, además de las iniciativas para el 'progreso' económico autosostenible y el aprovechamiento de la biodiversidad del Pacífico, el discurso del desarrollo incluyó "un componente social" dirigido a la formación de liderazgos que promovieran la participación comunitaria (Agudelo, 2005: 88). De igual forma, la descentralización fue importante en su acción de poner en el mapa a los "nuevos actores políticos y sociales" a nivel local y regional (Agudelo, 2005:89), en contraste con los lugares tradicionales de poder desde los cuales se habían dirigido las políticas de la nación. Esto es, en otras palabras, lo que más tarde tomaría forma como democracia participativa.

De igual manera hay que señalar, como nos recuerda Agudelo, que la adscripción política de la mayor parte del Pacífico fue y sigue siendo al partido Liberal, por su oposición histórica al esclavismo y por el ideario de libertades que pregonaba (Agudelo, 2005: 111). Igualmente, el autor resalta que la permanencia de dicha filiación política está imbuida de prácticas clientelistas y su perpetuación responde, sobre todo, a tradiciones familiares. No obstante, Agudelo también nota que a partir de finales de 1970 se ve una insurgencia de movimientos cívicos en la región, articulados a los partidos tradicionales, como resultado de las movilizaciones de los 'nuevos' movimientos sociales (Agudelo, 2005: 159) y manifestaciones estudiantiles. Estas organizaciones políticas cobran fuerza, alimentadas por las luchas sociales en otros lugares como Estados Unidos y África con 
el movimiento anti-apartheid. Surgen así en 1982 y 1986 los movimientos Cimarrón ${ }^{2}$ y $\mathrm{ACIA}^{3}$, respectivamente; considerados, entre otros, los más trascendentes y de mayor continuidad, funcionando hasta hoy.

La interacción de los factores referidos, en conjunto con el clima del país, contribuyeron a crear el caldo de cultivo en el que se dio la ANC, de la cual se desprendería el Artículo Transitorio 55 y posteriormente la Ley de Comunidades Negras. Con todo, las iniciativas sociales afrocolombianas no pudieron organizar una candidatura unificada para las elecciones de la ANC, por lo que se aliaron con los representantes indígenas Lorenzo Muelas y Francisco Rojas Birry, para asegurar su presencia, así fuera indirecta, en el proceso.

Por último, pero no menos importante, se debe distinguir la influencia de la Academia en la escritura de la Ley 70, específicamente. En Colombia el discurso académico, y la antropología especialmente, estaba marcado por las corrientes indigenistas y ecológicas que estuvieron en boga desde 1960, según recopilaciones de Sherry Ortner (1984). Lo anterior, entonces, contribuyó a darle a la Ley de Negritudes su carácter etnicista, indigenista y exclusivo que reivindica la multiculturalidad sólo desde lo étnico.

\section{Constitución de 1991 y Ley 70 de 1993}

Gracias a la gestión de los representantes indígenas en la ANC y a las campañas de presión ${ }^{4}$ impulsadas por movimientos negros, se logró la inclusión del Artículo Transitorio 55 (AT55) a la Constitución de 1991. Este artículo delega en una comisión especial, con participación de representantes de las comunidades involucradas, la creación de una ley que reconozca la titulación colectiva de tierras baldías ribereñas en la Cuenca del Pacífico, aplicable a zonas de condiciones similares, a comunidades negras que mantengan prácticas tradicionales de producción. La misma ley pretende "establecer mecanismos para la protección de la identidad cultural y los derechos de estas comunidades, y para el fomento de su desarrollo económico y social" (AT55).

\footnotetext{
2 Movimiento Nacional por los Derechos Humanos de las Comunidades Negras en Colombia, Cimarrón.

3 Asociación Campesina Integral del Atrato.

4 Entre las que se cuentan "el telegrama negro", la ocupación de la Catedral de Quibdó y la toma de la embajada de Haití.
} 
Aunque la aprobación del AT55 y su posterior desarrollo por la Ley 70 de 1993, fueron un triunfo para las comunidades rurales afrocolombianas, aún quedan varias preguntas abiertas. Teniendo en cuenta, como dice Agudelo, que el referente que tenían los grupos étnicos afro para las reivindicaciones de sus derechos y espacio político eran justamente los logros alcanzados por los indígenas en la ANC (Agudelo, 2005), es posible comprender una cierta decepción al no alcanzar los niveles de autonomía que sí tuvieron los otros. Las Entidades Territoriales Indígenas (ETIS), definidas, creadas y reglamentadas en los Artículos 286, 329 y 330 de la Constitución, tienen autonomía territorial, política y económica mientras que la titulación colectiva de tierras no goza de este beneficio. Sin embargo, la Ley 70 promovió la creación de una figura indispensable para el proceso de percepción y manejo de tierras colectivas: los Consejos Comunitarios, que serían reglamentados mediante el Decreto 1745 de 1995. "A pesar de que constitucionalmente a los Consejos Comunitarios no se les reconoce jurisdicción especial sí se les reconoce el pluralismo jurídico (diversidad de derechos) y con ello la existencia de sistemas jurídicos propios" (Moreno, 2010: 122). Se configura, entonces, como una estrategia de participación política de la comunidad dentro de sí misma.

Pasando a otros ejemplos, el Artículo 1 de la Ley 70 abre posibilidades y a la vez deja inquietudes. Después de mencionar lo referente a los derechos territoriales colectivos, el documento incluye otro propósito: "establecer mecanismos para la protección de la identidad cultural y de los derechos de las comunidades negras de Colombia como grupo étnico, y el fomento de su derecho económico y social, con el fin de garantizar que estas comunidades obtengan igualdad de oportunidades frente al resto de la sociedad colombiana” (Ley 70 de 1993). Aquí se hace, entonces, un intento de integrar la totalidad de los afrocolombianos en un discurso inclusivo y diferenciador. Sin embargo, no todos, más bien muy pocos, los habitantes pertenecientes a poblaciones urbanas se verán identificados con la pertenencia a grupos étnicos y comunidades en la forma en que son definidas en la legislación. De ésta manera, una norma que pretende reivindicar los derechos de un sector minoritario, termina siendo excluyente para una parte del mismo; tal vez por las mismas circunstancias históricas que han rodeado la lucha política por la diferenciación en el país. La Ley 70 "pone el acento en una identidad negra rural y del Pacífico y este factor (...) hace sentir ajenos a la mayoría de pobladores negros colombianos" 
(Agudelo, 2005: 207). Como se puede imaginar, las articulaciones de pertenencias y los reclamos políticos no van a ser los mismos para alguien inscrito en un contexto rural, que para otro sumergido en uno urbano ${ }^{5}$. Es evidente que la mal llamada Ley de Negritudes fue "imaginada para y desde el Pacífico colombiano (y más específicamente) pensada para poblaciones ribereñas" (Restrepo, 2011) ${ }^{6}$.

Se comprende, entonces, que lo negro como una alteridad que pedía reivindicaciones políticas y sociales, fue construido a partir de la misma redacción de la Ley de Comunidades Negras. "Lo negro" se configuró con unos límites y características específicas, haciendo énfasis en lo rural y, dentro de ello, en lo ribereño. Esto se puede rastrear, tal vez, a una asimilación con la legislación y la situación indígena que sí estuvo muy presente en la ANC. De cualquier forma, la delimitación de los sujetos a quienes se aplicaría la Ley 70 fue hecha arbitrariamente y conducida, sobre todo, por las corrientes académicas imperantes que señalé antes. Como bien señala Restrepo: "ser negro en términos étnicos fue una invención de los 90. La conformación de gente negra como grupo étnico tiene implicaciones politicas y en las subjetividades de la gente" (Restrepo, 2011).

Siguiendo la línea de las propuestas de integración a nivel nacional, en el mismo año de la aprobación de la Ley 70, se creó el Proceso de Comunidades Negras (PCN): un movimiento político nacional sucesor de la Coordinadora Nacional de Comunidades Negras ${ }^{7}$ que agrupó en un principio a todos los integrantes, menos a los chocoanos, del proceso de coordinación para la concreción de la Ley mencionada (Agudelo, 2005). Hoy, además de las líneas de procesos de autonomía, fortalecimiento de la identidad cultural y los derechos y manejo de recursos, el PCN ha incluido a su discurso elementos referentes a la prevención de violencia en las comunidades en el marco del conflicto armado, con especial énfasis en la visibilización del desplazamiento y la situación de las mujeres y los jóvenes. En contraste con los procesos de movilización de surgimiento popular y voluntario, dentro de la misma ley en el Artículo 66 del Capítulo VIII se establece por iniciativa estatal (jalonada por el pro-

$5 \quad$ Hago esta diferenciación sabiendo que hay espacios en dónde las fronteras entre lo rural y lo urbano se vuelven difusas.

6 La información citada hace parte de un conversatorio sobre implicaciones de la Ley 70 en el Pacífico Colombiano, que impartió Eduardo Restrepo el 11 de marzo de 2011.

7 "Espacio federado de las organizaciones [que] (...) definió como eje principal de su lucha la exigencia del derecho al territorio para las poblaciones negras del Pacífico" (Agudelo, 2005: 180). 
ceso histórico y político precedente) "la circunscripción especial para elegir a dos miembros de las comunidades negras del país asegurando su participación en la Cámara de Representantes" (Ley 70 de 1993). Esto de conformidad con los Artículos 171 y 176, que fijan respectivamente, dos curules de circunscripción especial por comunidades indígenas para el Senado de la República y una para la Cámara. Esta reglamentación se hace con miras a darle representatividad a las minorías étnicas dentro del Sistema Legislativo, sin embargo, cabe preguntarse si dos o tres congresistas o senadores, pueden representar plenamente los intereses de un sector no despreciable de la población.

Agudelo poner de manifiesto la pregunta por la representatividad y la ejemplifica con el caso de las elecciones de 2001, en las cuales las curules pertenecientes a las poblaciones afrocolombianas fueron ocupadas dos ex-deportistas: María Isabel Urrutia y Willington Ortiz. No es difícil darse cuenta que éstos perfiles no son exactamente los que representen la identidad cultural, la cosmovisión y ni siquiera las condiciones de vida de las comunidades rurales ribereñas de la Cuenca del Pacífico. En adición, sus candidaturas hicieron más ahínco en favorecer al "deporte y los sectores populares sin que hubiera una alusión clara a las problemáticas de las poblaciones negras” (Agudelo, 2005: 204). Actualmente, las críticas -ahora desde los medios de comunicación y la opinión generalse vuelcan a tildar la circunscripción especial para las negritudes como un atajo fácil para llegar a la Cámara, impulsado por prácticas clientelistas y partidos de dudosa reputación.

Según el informe realizado por el Movimiento de Observación Electoral (MOE) sobre los resultados de las circunscripciones especiales del Congreso en las elecciones de 2010, para las Comunidades Negras se presentaron 67 listas de diferentes partidos, fundaciones y movimientos, para un total de 170 candidatos”. Del total de 10'502.122 de votos válidos para la Cámara de Representantes, sólo 549.061 fueron para la circunscripción especial de negritudes y un poco más de la quinta parte fueron votos en blanco. Los candidatos elegidos fueron Yahir Fernando Acuña y Heriberto Arrechea con el 9.3\% y $5.8 \%$ de los votos totales de circunscripción, respectivamente. Pertenecen, el primero a la Asociación de Afrocolombianos para la Vivienda, Deporte, Educación y Salud AFROVIDES, y el segundo al Movimiento Popular Unido ${ }^{9}$. De Acuña no se conoce

8 Norma declarada inexequible por la Corte Constitucional en 1996 y restablecida para las elecciones de 2001.

9 Mismo partido al que pertenecía María Isabel Urrutia cuando fue elegida. 
trabajo pasado con las comunidades afro; su inscripción se dio a último minuto y ha sido severamente cuestionada. Sobre Arrechea también caen críticas de amistades dudosas. Sus propuestas parecen estar enfocadas al bienestar de las comunidades negras, más es muy pronto para juzgar los resultados. En suma, el MOE reconoce que no hay partidos ni tendencias consolidadas y presume que esto se debe a los blandos requisitos para la inscripción de los candidatos de las comunidades negras para la circunscripción especial ${ }^{10}$. Esta situación no ayuda tampoco a los propósitos representativos y reivindicativos en el marco de los cuales se desarrolló la Ley 70 de 1993.

Otro mecanismo implementado por la Constitución de 1991 fue la consulta previa. En un principio adoptado como parte del Convenio 169 de la OIT aprobado mediante la Ley 21 de 1991, fue reglamentada más tarde por el Decreto 1320 de 1998. "El desarrollo y la implementación de la consulta previa debe entenderse como un derecho vinculado a la participación" (Mosquera, 2010: 160). La consulta previa le permite a las comunidades, indígenas y negras, informarse e involucrarse en la toma de decisiones de proyectos que las impacten de alguna manera. Sin embargo, en el tiempo que ha estado funcionando esta figura, se dan denunciado, como lo hizo la periodista Laura Rico Piñeres en el 2009, varias infracciones que van desde ignorar la medida completamente, pasando por no verificar si el territorio que será intervenido está habitado por comunidades étnicas, hasta viciar las reuniones concertadas o realizarlas después de empezado el proceso invasivo.

Ahora quisiera hacer referencia a otro de los factores importantes que se cruzan en la agenda de participación política propuesta por la Ley de Comunidades Negras y que, por lo demás, desgraciadamente es un lugar común para todo el país. Agudelo anota que hasta mediados de los 90 el Pacífico se mantenía como una 'zona de paz' con respecto al conflicto armado a nivel nacional (Agudelo, 2005). Sin embargo, después del "proceso organizativo de las comunidades y la aplicación de la Ley 70 de 1993, [...] las tensiones alrededor de los conflictos por territorios toman nuevas dimensiones" (Agudelo, 2005: 211). En este periodo empiezan a cobrar importancia situaciones como: conflictos interétnicos, así como entre partidarios y detractores de la Ley 70, entre pobladores negros y empresas extractivas y entre fuerzas guerrilleras y paramilitares. "La ubicación geográfica estratégica para los 
actores armados, las perspectivas de macroproyectos de desarrollo, el potencial de recursos naturales explotables son, entre otros, aspectos que explican el porqué de la transformación de la región en un escenario principal del conflicto" (Agudelo, 2005: 22). El conflicto armado se agudiza en 1995 y desemboca en el desplazamiento forzado de muchas comunidades rurales por encontrarse en medio del enfrentamiento entre guerrillas y paramilitares, quienes, aprovechando la poca presencia del Estado y la fuerza pública, empezaron a tomar acciones radicales y violentas para posesionarse de la región. Agudelo ilustra: "en el marco de (la) ofensiva contreinsurgente se (produce) entre diciembre de 1996 y febrero de 1997, el desplazamiento forzado de aproximadamente 15.000 personas en el área del Bajo Atrato" (Agudelo, 2005: 217); tendencia que se continua en la región por temor a futuros enfrentamientos. En este proceder, las titulaciones de tierras de las comunidades interferían en los planes expansionistas de los grupos armados y, además, de las grandes compañías extractivas. El autor se refiere también al caso de la parte norte del río Truandó, en dónde se entregó el título colectivo de tierras a una comunidad en su mayor parte desplazada y que contaba con líderes comunitarios asesinados por los paramilitares (Agudelo, 2005: 218).

\section{Conclusiones}

Con los contrastes señalados entre los propósitos de la Ley de Comunidades Negras y las prácticas de participación que han creado y a las que se han adscrito dichas comunidades, me atrevo a sugerir una conclusión que puede parece obvia. La Ley 70 no ha cumplido con su propósito de "establecer mecanismos para la protección de la identidad cultural y de los derechos de las comunidades negras de Colombia como grupo étnico, y el fomento de su derecho económico y social, con el fin de garantizar que estas comunidades obtengan condiciones reales de igualdad de oportunidades frente al resto de la sociedad colombiana" (Ley 70 de 1993). Lo anterior es posible rastrearlo en la incidencia que la violencia ha tenido en la región Pacífica desde mediados de 1990 y a la dificultad que tiene la Ley de manifestar la heterogeneidad de las mismas comunidades.

En primer lugar la violencia, llegada recientemente al Pacífico, tiene efectos negativos para el desarrollo correspondiente de los procesos organizativos que acompañan la titulación colectiva de tierras legislada por la Ley de Negritudes. De esta forma se aminoran también las oportunidades de participación política, fomentada por los Consejos 
Comunitarios por ejemplo, de las que se supone deberían privilegiarse las comunidades por ser consideradas una minoría étnica. Además, ésta situación afecta directamente las condiciones de vida de los pobladores que son desplazados de sus tierras y que como tales, se convierten -idealmente- en sujetos de políticas proteccionistas más que en gestores de movimientos que abogue por su inclusión en la sociedad.

En segundo lugar, y lo que me parece más importante, está la cuestión de la creación y etnización de lo negro como una entidad homogénea delimitada y excluyente. La Ley 70 falla en percibir y materializar la heterogeneidad de las comunidades negras, tanto en contextos rurales como urbanos y dentro de estos mismos. Con esta imagen totalizadora de lo que considera afrocolombiano, la misma legislación limita los lugares de participación de las comunidades pues invisibiliza la mayor porción de negritudes del país: las ciudades. La Ley pretende definir quién es negro y sólo en virtud de dicha definición se articulan las posibilidades de participación. Los problemas de representatividad, como se evidencia en el caso de la circunscripción especial para comunidades negras, están ligados también, en parte, a la construcción de la etnicidad negra. Combinado esto con su blanda legislación, resulta en una situación en la que no se ven representados los habitantes rurales ni los urbanos. En cuanto a esta clasificación, sobre la que me he apoyado antes, cabe dejar claro que es en sí misma simplificadora, pues dentro de los contextos rurales y urbanos se pueden encontrar diversas clasificaciones que pueden o no coincidir con la etnización de lo negro.

Para asegurar una participación equitativa y una representación verdadera, me parece prudente que se emprenda un proceso de revisión de las 'categorías' vigentes de lo que se considera negro y poder plantear modificaciones destinadas a visibilizar la heterogeneidad de lo rural y lo urbano, incluso dentro de ellos mismos. Además hay que ser capaces de dar cuenta de los cambios históricos, como la violencia, que afectan las condiciones de vida y las posiciones de sujeto de las personas y conforme a estas dinámicas, proponer una legislación que sea acorde a ellas. A dos décadas de la promulgación de la Constitución de 1991, su cambio de contenido sigue siendo visto como un atentado al orden político del país, cuando debería entenderse como consecuencia de las circunstancias históricas y la agencia de los individuos, quienes constantemente están transformando la nación. 


\section{Bibliografía}

AGUDELO, Carlos (2005). Retos del multiculturalismo en Colombia: politica y poblaciones negras. Medellín: La Carreta Social, 2005.

ARRECHEA, Heriberto (2011). “Contenido programático de la campaña”. Página oficial. Consultado el 28 de marzo de 2011, desde: http:/www.heribertoarrechea.com/ index.php?option $=$ com_content $\&$ view $=$ article $\&$ id $=45 \&$ Itemid $=59$

BONILLA, Daniel (2006). La Constitución multicultural. Bogotá: Pontificia Universidad Javeriana

CASTIBLANCO, Esteban (2011). "Resultados de las circunscripciones especiales del Congreso en las elecciones de 2010". En Impactos de los Aspectos Socioeconómicos en la Democracia Local y Regional, Movimiento de Observación Electoral. Fecha de actualización: abril 3 de 2011, desde: http://www.moe.org.co/webmoe/images/stories/ paper_resultados_2010.pdf

CONSTITUCIÓN POLÍTICA DE COLOMBIA. Bogotá: Panamericana, 1998.

GROS, Christian (2000). "Ser diferente por (para) ser moderno, o las paradojas de la identidad". En: Políticas de la etnicidad: identidad, estado y modernidad, pp. 97-115. Bogotá: ICANH.

HALL, Stuart (2010). "La cuestión de la identidad cultural”. En: Sin garantías: trayectorias y problemáticas en estudios culturales, pp. 363- 401. Instituto de Estudios Sociales y Culturales, Pensar. Universidad Javeriana

MORENO, Héctor, Alejandra Machado y Adolfo Rodríguez (2010). "El multiculturalismo en la Constitución de 1991: en el marco del bicentenario”, pp. 67-217. Cali: Programa Editorial Universidad del Valle

ORTNER, Sherry (1984). "La teoría antropológica desde los años sesenta”. En: Comparative Studies in Society and History, Vol. 26, núm. 1, Enero, pp. 126-166.

RENACIONESTE PROYECTO DE COMUNIDADES NEGRAS (2011). "Líneas de acción”. Página oficial, última actualización en el 2011. Consultado el 28 de marzo de 2011, desde: http://www.renacientes.org/index.php?option=com_content\&view=section \&layout=blog\&id=5\&Itemid $=155$

RESTREPO, Eduardo (2011). Implicaciones de la Ley 70 en el Pacífico colombiano. Conversatorio realizado en marzo 11 de 2011. Cali, Colombia: Universidad Icesi.

RICO, Laura (2009). Crónica: "La consulta previa: la farsa multicultural”. En: La 
Silla Vacía. Consultado el 28 de marzo de 2011, desde: http://www.lasillavacia.com/ historia/3201

SÁNCHEZ, Enrique (1993). "Derechos e identidad: los pueblos indígenas y negros en la constitución política de Colombia de 1991", capítulos 4 y 6. Bogotá: Disloque editores

TURCIOS, Luz Elena (2011). "Sucreño gana curul de las negritudes". En: El Universal, 17 Marzo 2010. Consultado el 28 de marzo de 2011, desde: http://www.eluniversal. com.co/monteria-ysincelejo/local/sucreno-gana-curul-de-las-negritudes

WILLS, María Emma (1999) "De la nación católica a la nación multicultural: rupturas y desafíos”. En: Museo, memoria y nación, núm. 4, pp. 387-411 Cátedra Anual de Historia "Ernesto Restrepo Tirado".

Decritos, leyes y jurisprudencia

Decreto 1745 de 1995.

Ley 70 de 1993. 